\title{
AUTOMATIC OBJECT DETECTION BASED ON ADAPTIVE BACKGROUND SUBTRACTION USING SYMMETRIC ALPHA STABLE DISTRIBUTION
}

\author{
Harish Bhaskar $^{1}$, Lyudmila Mihaylova ${ }^{1}$ and Alin Achim ${ }^{2}$ \\ ${ }^{1}$ Department of Communication Systems, Lancaster University, Lancaster, UK \\ ${ }^{2}$ Department of Electrical and Electronic Engineering, Bristol University, UK \\ \{h.bhaskar,mila.mihaylova\}@lancaster.ac.uk,alin.achim@bristol.ac.uk
}

Keywords: automatic object detection, tracking, background subtraction, alpha stable distribution, video sequences

\begin{abstract}
Automatic detection of objects is critical to video tracking systems. One of the simplest techniques for detection is background subtraction (BS). BS refers to the process of segmenting moving regions from image sequences. The BS process involves building a model of the background and extracting regions of the foreground (moving objects). In this paper, we propose an extended cluster BS (CBS) technique based on symmetric alpha stable $(S \alpha S)$ distributions. The developed method functions at cluster-level as against the traditional pixel-level BS methods. An iterative self-adaptive mechanism is presented that allows automated learning of the distribution of the model parameters. The results for the CBS $S \alpha S$ algorithm on real video sequences show improvement compared with a CBS using a Gaussian mixture model.
\end{abstract}

\section{Introduction}

Detection is an inherent part of any efficient tracking algorithm. The detection process helps identifying and localising moving objects within any scene. The simplest way of accomplishing detection is through building a representation of the background and comparing each new frame with this representation. This procedure is known as background subtraction (BS) [3]. Some of the popular techniques for BS include mixture of Gaussians [24], kernel density estima- tion [7], colour and gradient cues [9], high level region analysis [21], Kalman filtering [23], hidden Markov models [20], and Markov random fields [14]. The general idea behind the aforementioned probabilistic techniques is to consider each pixel as a random variable and to represent each pixel of the image by means of its probability density function (PDF). A pixel from a new image is classified as background depending on the pixel PDF. In the last several years, a number of different BS techniques have been proposed in the literature. The very basic BS techniques are detecting the foreground objects as the difference between two subsequent video frames, operate at a pixel level and are applicable to static backgrounds. If the PDF of the pixel is bigger than a threshold, the pixel is classified as a background.

Though the generic BS method is simple to understand and implement, the disadvantages of the frame difference $\mathrm{BS}$ is that it does not provide a mechanism for choosing the parameters, such as the detection threshold, and its inability to cope with multi-modal backgrounds. In an attempt to address the inadequacies of the basic methods, the idea of modeling the background as the average or median of pixels from the previous frames was proposed in [11]. Though these techniques were more robust than the basic methods, the problems of choosing threshold and multi-modal distributions remained.

One of the important techniques proposed to cope with multi-model background distribution and to update the detection threshold was based on Gaussian mixture models (GMMs). The model proposed by $[24,15]$ describes each pixel as a mixture of Gaussians and relies on an on-line distribution to update this model. The 
larger Gaussian components correspond to the background and this is used to generate the background model. In [1] a cluster-based GMM is developed and its better performance compared with the pixel-level GMM [24] is demonstrated.

In contrast to the GMM, kernel density estimators (KDEs) [7, 6] evaluate the histogram of the $n$ most recent pixel values, each smoothed with a Gaussian kernel, and represent the background PDF in this way. A disadvantage of this approach is that it has high memory requirements (proportional to the number of pixels times the size of a frame). The KDE of Elgammal et al. [7] relies on a Gaussian kernel function which requires $N$ Gaussian components where typically $N$ is between 50 and 100 . This requires more computations compared with the GMM of Stauffer and Grimpson [19] which relies on a small number of components (typically between three and five).

Some of the other recently developed BS techniques include the mean-shift estimators. The mean-shift estimators [8] are gradient methods. Their standard iterative implementation is slow and with high memory requirements. Some improvements include computational optimisations, or using it only for detecting the background PDF modes at the initialisation step. However, most of these techniques have limitations and cannot handle dynamic changes of the background, e.g., gradual or sudden (e.g., moving clouds), motion changes including camera oscillations and high frequency background objects (tree branches, sea waves, etc.) and changes in the background geometry (such as parked cars) [3].

An algorithm for background modeling and subtraction based on Cauchy statistical distribution is presented in [13]. It is shown that the Cauchy distribution with its heavier tail is able to represent the tails of the histogram in a better way in comparison with the Gaussian distribution. The model has been shown to be robust and adaptive to dynamic changes of the background scene such as moving trees, rain, camera motion and local/global illumination changes.

In this paper, we propose a novel technique of cluster BS based on symmetric alpha stable (CBS$S \alpha S$ ) distributions. Whilst the cluster background subtraction (CBS) method helps reducing the clutter, the symmetric alpha stable $(S \alpha S)$ distributions help handling dynamic illumination changes in a scene and model moving backgrounds in a better way due to the heavy tail. It is also important to note that results of modeling the background of a moving image sequence can be best obtained while operating with arbitrary values of the characteristic exponent parameter of the $S \alpha S$ distribution, rather than fixed values such as the Gaussian or Cauchy case. By automatically estimating the parameters of the alpha stable distribution, the PDF of clusters of pixels can be faithfully represented and a reliable model of the background can be obtained.

The remaining part of the paper is organised as follows. Section 2 presents the Bayesian formulation of BS problem. A description of different distributions in the context of BS is given in the subsequent section of the paper. Experimental results are given in Section 4. Comparative results obtained with a cluster-based GMM BS are presented. Finally, we present some conclusive remarks and directions of future work in Section 5 .

\section{Problem Formulation}

The problem of cluster background subtraction (CBS) involves a decision whether a cluster of pixels belongs to the background ( $b G$ ) or foreground $(f G)$ object based on the ratio of probability density functions:

$$
\frac{p\left(b G \mid \boldsymbol{c}_{k}^{i}\right)}{p\left(f G \mid \boldsymbol{c}_{k}^{i}\right)}=\frac{p\left(\boldsymbol{c}_{k}^{i} \mid b G\right) p(b G)}{p\left(\boldsymbol{c}_{k}^{i} \mid f G\right) p(f G)}
$$

where, the vector $c_{k}^{i}=\left(c_{1, k}^{i}, \ldots, c_{\ell, k}^{i}\right)$ characterises the $i$-th cluster $(0 \leq i \leq q)$ at time instant $k$ (and current image), containing $\ell$ number of pixels such that $[\mathrm{Im}]_{k}=\left[\boldsymbol{c}_{k}^{1}, \ldots, \boldsymbol{c}_{k}^{q}\right]$ is the whole image; $p\left(b G \mid c_{k}^{i}\right)$ is the PDF of the background, subtracted using a certain feature (typically colour or edges) of the cluster $\boldsymbol{c}_{k}^{i} ; p\left(f G \mid \boldsymbol{c}_{k}^{i}\right)$ is the PDF of the foreground on the same cluster $\boldsymbol{c}_{k}^{i} ; p\left(\boldsymbol{c}_{k}^{i} \mid b G\right)$ refers to the PDF model of the background and $p\left(\boldsymbol{c}_{k}^{i} \mid f G\right)$ is the appearance model of the foreground object. In our cluster BS technique the decision that a cluster belongs to a background is made if:

$$
p\left(\boldsymbol{c}_{k}^{i} \mid b G\right)>\text { threshold }\left(=\frac{p\left(\boldsymbol{c}_{k}^{i} \mid f G\right) p(f G)}{p(b G)}\right)
$$

Since the threshold is a scalar, the decision in (2) is made from the average of the distributions of all pixels within the cluster $\boldsymbol{c}_{k}^{i}$. 


\section{Background Modeling}

Most of the existing BS techniques such as $[7,24,19,3]$ operate at pixel level in contract to the algorithm proposed here, which operates at cluster level. The appearance of the foreground, characterised by the PDF $p\left(\boldsymbol{c}_{k}^{i} \mid f G\right)$, is assumed uniform. The background model represented as $p\left(\boldsymbol{c}_{k}^{i} \mid b G\right)$ is estimated from a training set $\Re$ which is a rolling collection of images over a specific update time $T$. The time $T$ is crucial since its update determines the model ability to adapt to illumination changes and to handle appearances and disappearances of objects in a scene. If the frame rate is known, the time period $T$ can be adapted: $T=\frac{N}{f p s}$, e.g., as a ratio between the number $N$ of frames obtained through the online process and the frame rate, $f p s$, frames per second. At time instant $k$ we have $\Re_{k}=\left\{\boldsymbol{c}_{k}^{i}, \ldots, \boldsymbol{c}_{k-T}^{i}\right\}$.

Every cluster $c_{k}^{i}, \quad(0 \leq i \leq q)$ at time instant $k$ is generated using a colour clustering mechanism of the nearest neighbour approach [22], although other techniques can be used. The aim of the clustering process is to separate data according to certain similarities. Clustering is carried out based on the hue, value, saturation (HSV) colour model due to its inherent ability to cope with illumination changes. Constraints such as spatial distance, hue difference and brightness changes are imposed on the model.

\subsection{The Proposed CBS- $S \alpha S$ Model}

We assume that a $S \alpha S$ distribution represents the PDF of the foreground and background. The image is clustered according to the nearest neighbour approach [5]. For each cluster the parameters of the $S \alpha S$ distribution are evaluated with an iterative technique. Then a decision is taken whether a cluster belongs to the background or to the foreground based on the colour histogram of the cluster. In the subsection below, we describe in detail the algorithm for cluster background update using the parameters of the $S \alpha S$ distribution.

\subsubsection{Alpha Stable Distribution}

The appeal for $S \alpha S$ distributions as a statistical model for signals derives from some important theoretical and empirical reasons. Generally, there is no a closed-form expression for the PDF of $S \alpha S$ distributions. Hence, the most convenient way of defining them is by means of their characteristic function

$$
\varphi(\boldsymbol{c})=\exp \left(j \delta \boldsymbol{c}-\gamma|\boldsymbol{c}|^{\alpha}\right)
$$

where

- $\alpha$ is the characteristic exponent parameter, with values $0<\alpha \leq 2$. It is arguably the most important parameter as it determines the shape of the distribution. It controls the heaviness of the tails of the density function. A small positive value of $\alpha$ indicates severe impulsiveness, and thus tails are heavier, while a value of $\alpha$ close to 2 indicates more Gaussian type of behaviour. A value of $\alpha=1$ corresponds to Cauchy distribution.

- $\delta$ is the location parameter $(-\infty<\delta<\infty)$. It corresponds to the mean for $1<\alpha \leq 2$, and to the median for $0<\alpha \leq 1$.

- $\gamma$ is the dispersion parameter $(\gamma>0)$, which determines the spread of the density around the location parameter. It behaves in a similar way to the variance of the Gaussian density, and it is, in fact, equal to half of the variance when $\alpha=2$, for the Gaussian case.

A $S \alpha S$ distribution characterised by the above three parameters is denoted as $\mathcal{S}(\alpha, \gamma, \delta)$.

\subsubsection{Gaussian Distribution}

The case $\alpha=2$ corresponds to the Gaussian distribution. The PDF has the form

$$
f_{\alpha=2}(\gamma, \delta ; \boldsymbol{c})=\frac{1}{\sqrt{4 \pi \gamma}} \exp \left\{-\frac{(\boldsymbol{c}-\delta)^{2}}{4 \gamma}\right\}
$$

\subsubsection{Cauchy Distribution}

The case where $\alpha=1$ corresponds to the Cauchy distribution. The PDF is given by

$$
f_{\alpha=1}(\gamma, \delta ; \boldsymbol{c})=\frac{\gamma}{\pi\left[\gamma^{2}+(\boldsymbol{c}-\delta)^{2}\right]}
$$

The smaller the characteristic exponent $\alpha$ is, the heavier the tails of the symmetric $\alpha$ stable density. This implies that random variables following symmetric $\alpha$ stable distributions with small characteristic exponents are highly impulsive. In fact, no closed-form expressions for the general symmetric $\alpha$ stable PDF are known except for the Gaussian and the Cauchy members. General $\alpha$ stable density members do not possess finite second or higher moments [17]. In particular, the dispersion of the stable distribution with $\alpha<2$ 
does not exist, making it meaningless as a measure of dispersion. However, the dispersion of a stable random variable plays an analogous role to the variance. The larger the dispersion of an $\alpha$ stable variable is, the more spread it is around its location parameter [18].

The BS schemes based on Cauchy distributions have more advantages than the Gaussian distribution based methods. The heavier tails of the Cauchy distribution represent in a better way the tails of the histograms than the Gaussian case. Additionally, the Cauchy without exponential operation is more cost effective in comparison to the Gaussians.

\subsubsection{Iterative Parameter Estimation}

The most important parameters of a $S \alpha S$ distribution are the characteristic exponent $\alpha$ and the dispersion parameter $\gamma$. The location parameter $\delta$ can often be assumed to be zero, i.e., the measurements are normalised with respect to the origin. Several methods for estimating these parameters have been introduced $[12,10]$.

In this paper, we employ an iterative $S \alpha S$ distribution parameter estimation technique. At any time instant $k$, let $\Re$ denote all the existing $\ell$ pixels under consideration (actually $\Re$ is the rolling set of the considered images). The update of the parameter estimates $\boldsymbol{\alpha}_{(1, k)}, \boldsymbol{\alpha}_{(2, k)}, \ldots, \boldsymbol{\alpha}_{(M, k)}$ and $\gamma_{(1, k)}, \boldsymbol{\gamma}_{(2, k)}, \ldots, \boldsymbol{\gamma}_{(M, k)}$ at time instant $k$ is performed from the estimates $\boldsymbol{\alpha}_{(1, k-1)}, \boldsymbol{\alpha}_{(2, k-1)}, \ldots, \boldsymbol{\alpha}_{(M, k-1)} \quad$ and $\gamma_{(1, k-1)}, \gamma_{(2, k-1)}, \ldots, \gamma_{(M, k-1)}$ at previous time instant $k-1$.

If $\Re$ is a real $S \alpha S$ random variable, i.e., if $\Re$ is assumed as a $S \alpha S$ time series of dependent or independent samples, then its $p$-th order moment satisfies the following relation. This implies that $V=\log |\Re|$ corresponds to a $\log |S \alpha S|$ process with $\boldsymbol{\mu}$ and $\sigma$ representing the mean and variance of the $\Re$ samples. The estimates of the mean and the variances, $\widetilde{\boldsymbol{\mu}}_{1, k}, \ldots, \widetilde{\boldsymbol{\mu}}_{M, k}$ and $\widetilde{\sigma}_{1, k}^{2}, \ldots, \widetilde{\sigma}_{M, k}^{2}$, respectively of the $\Re$ samples can be represented as in [25] using the following update equations,

$$
\begin{gathered}
\widetilde{w}_{m, k+1}=\widetilde{w}_{m, k}+\frac{1}{T_{k}}\left(o_{m, k}-\widetilde{w}_{m, k}\right), \\
\widetilde{\boldsymbol{\mu}}_{m, k+1}=\widetilde{\boldsymbol{\mu}}_{m, k}+o_{m, k}\left(\frac{1}{T_{k} \widetilde{w}_{m, k}}\right) \boldsymbol{\delta}_{m, k}, \\
\widetilde{\sigma}_{m, k+1}^{2}=\widetilde{\sigma}_{m, k}^{2}+o_{m, k}\left(\frac{1}{T_{k} \widetilde{w}_{m, k}}\right)\left(\boldsymbol{\delta}_{m, k}^{\prime} \boldsymbol{\delta}_{m, k}-\sigma_{m, k}^{2}\right),
\end{gathered}
$$

where $\boldsymbol{\delta}_{m, k}=\boldsymbol{c}_{k}^{i}-\widetilde{\boldsymbol{\mu}}_{m, k},{ }^{\prime}$ denotes transpose, and $o_{m, k}$ refers to the ownership of the new cluster and defines the closeness of this cluster to a particular $S \alpha S$ component, and $m=1, \ldots, M$. The dispersion parameter $\gamma_{m, k}$ at any time instant $k$ can be updated iteratively by the equations [12]

$$
\begin{array}{r}
\frac{\log \boldsymbol{\gamma}_{m, k}}{\boldsymbol{\alpha}_{m, k}}=\frac{k-1}{k} \\
\left(\frac{C_{e}\left(1-\boldsymbol{\alpha}_{m, k-1}\right)+\log \boldsymbol{\gamma}_{m, k-1}}{\boldsymbol{\alpha}_{m, k-1}}\right) \\
+\frac{\boldsymbol{\mu}_{m, k}}{k}+C_{e}\left(1-\frac{1}{\boldsymbol{\alpha}_{m, k}}\right)
\end{array}
$$

and similarly the characteristic exponent $\alpha_{k}$ at time instant $k$ can be updated as in [12] using,

$$
\begin{array}{r}
\frac{\pi^{2}}{6}\left(\frac{1}{\boldsymbol{\alpha}_{m, k}^{2}}+\frac{1}{2}\right)= \\
\frac{k-1}{k^{2}}\left(\frac{C_{e}\left(1-\boldsymbol{\alpha}_{m, k-1}\right)+\log \gamma_{m, k-1}}{\boldsymbol{\alpha}_{m, k-1}}-\boldsymbol{\mu}_{m, k}\right)^{2}+ \\
\frac{\pi^{2}(k-1)}{6 k}\left(\frac{1}{\boldsymbol{\alpha}_{m, k-1}^{2}}+\frac{1}{2}\right)+\frac{1}{k} \sigma_{m, k},
\end{array}
$$

where $C_{e}=0.57721566 \ldots$ is the Euler constant. It is important to note that the accuracy of this parameter estimation technique increases with the increase in sample size. The ownership of any new cluster is set to 1 for "close" components and the others are set to zero. A cluster is close to a component iff the Mahalanobis distance between the component and the cluster centre is, e.g., less than 3. If there exist no "close" components, a component is generated with $\widetilde{w}_{m+1, k}=1 / T_{k}$, with an initial mean $\tilde{\boldsymbol{\mu}}_{0}$ and variance $\tilde{\sigma}_{0}^{2}$.

The model presents clustering of components and the background is approximated with the $B$ largest components,

$$
\begin{gathered}
\widetilde{p}\left(\boldsymbol{c}_{k}^{i} \mid \Re_{k}, b G\right) \sim \sum_{m=1}^{B} \widetilde{w}_{m, k} \varphi\left(\boldsymbol{\alpha}_{m, k}, \gamma_{m, k}\right), \\
B=\underset{b}{\operatorname{argmin}}\left(\sum_{m=1}^{b} \widetilde{w}_{m, k}>\left(1-c_{f}\right)\right),
\end{gathered}
$$

where $b$ is a variable defining the number of considered clusters, $c_{f}$ is the proportion of the data that belong to foreground objects without influencing the background model. The proportionality between the pixels belonging to the foreground to the pixels from the background is assumed constant in most adaptive models [24]. This assumption does not hold true when videos of objects 
are captured from a close proximity. In such circumstances, the proportion of pixels belonging to the objects of interest, i.e., the foreground pixels, are much higher than the background pixels. This ratio defining the percentage of foreground and background pixels can be updated from the training set as follows:

$$
c_{f}=\frac{\widetilde{p}\left(c_{k}^{i} \mid \Re_{k}, f G\right)}{\widetilde{p}\left(c_{k}^{i} \mid \Re_{k}, b G\right)} .
$$

\section{Results and Analysis}

The performance of the proposed symmetric alpha stable CBS (CBS-S $\alpha S$ ) technique will be illustrated over real video sequences from [16] and compared with the cluster-level GMM (CBS-
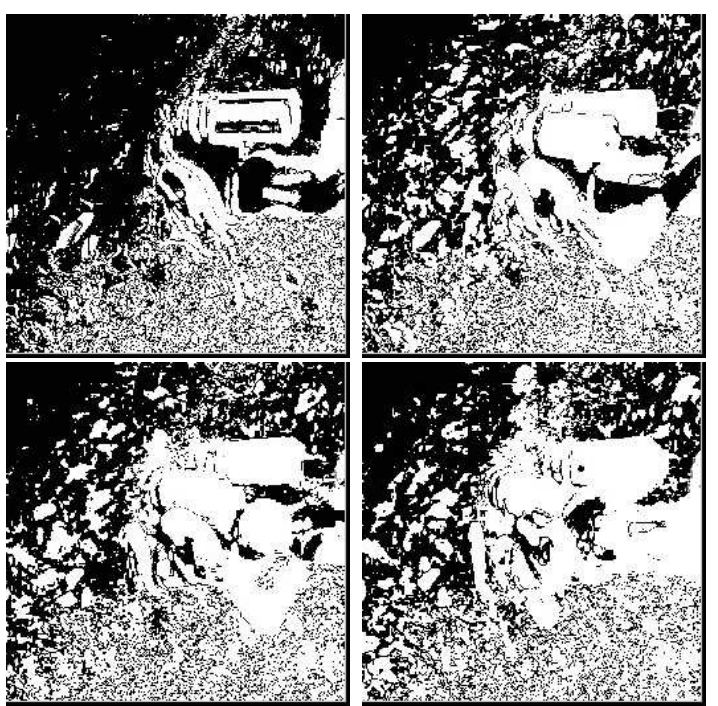

(a: Results from the CBS-GMM)
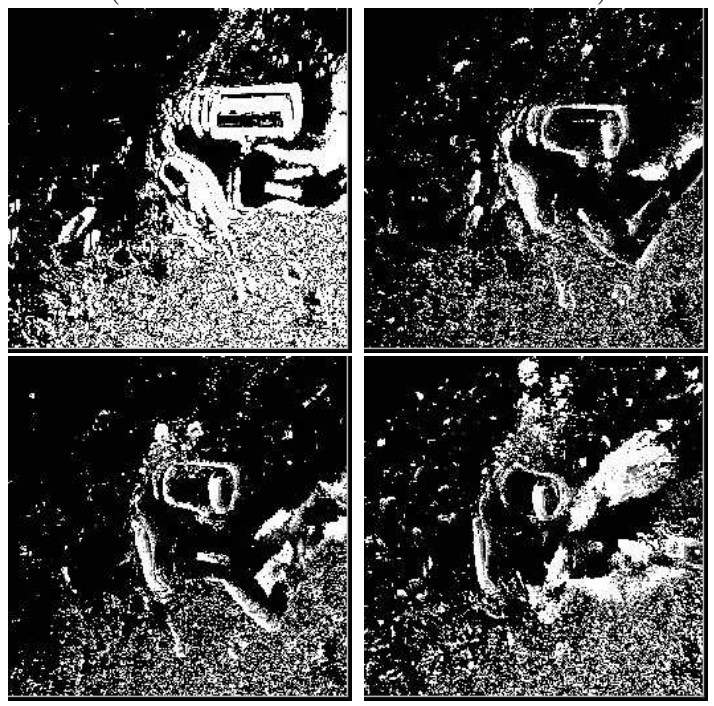

(b: Results from the CBS-S $\alpha S$ )

Figure 1: Results from the (a) CBS-GMM [1] and

(b) proposed CBS-S $\alpha S$ model on sequence 1
GMM) [1].
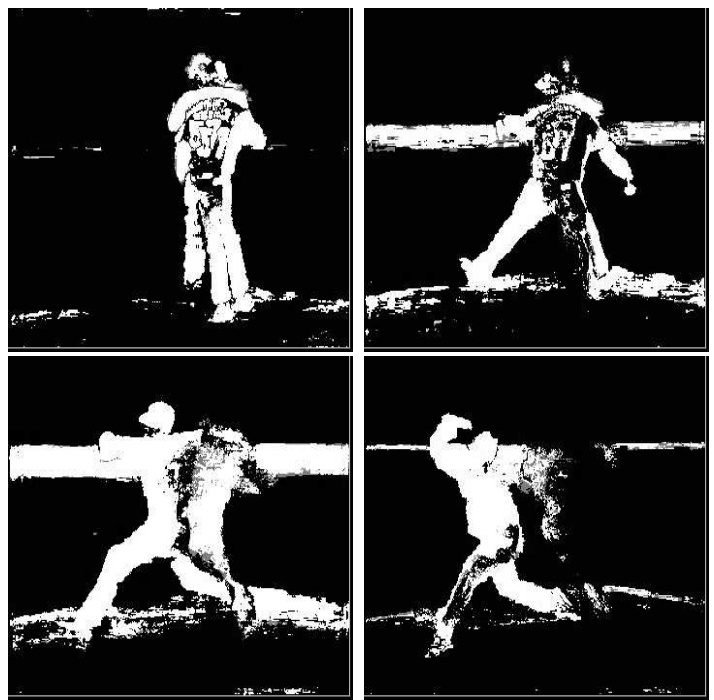

(a: Results from the CBS-GMM)
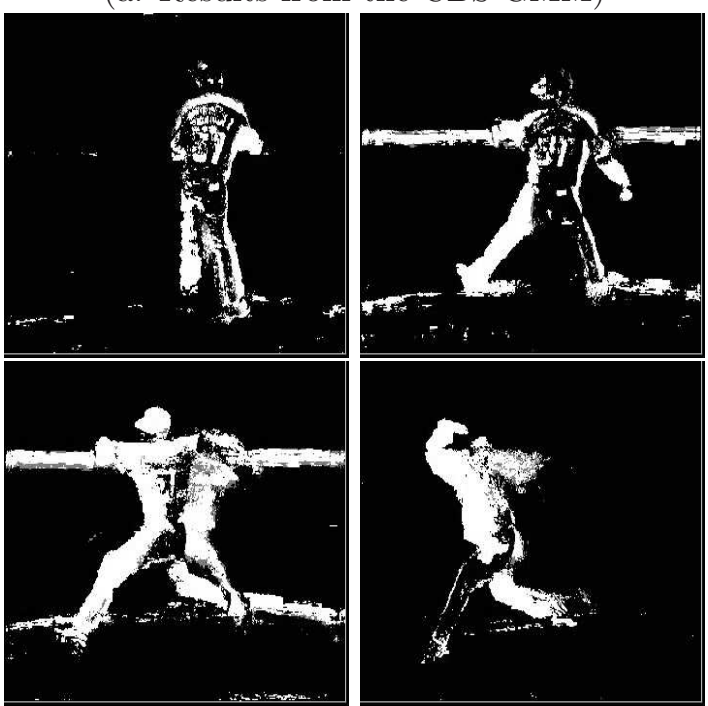

(b: Results from the CBS- $S \alpha S$ )

Figure 2: Results from the (a) CBS-GMM [1] and (b) proposed CBS- $S \alpha S$ model on sequence 2

It is evident from the results above, that the proposed CBS- $S \alpha S$ technique gives results with less clutter than the CBS-GMM method. The techniques are also compared using quantitative measurements such as recall and precision. Recall and precision measures quantify how well an algorithm matches the ground truth [2]. Recall [4] is calculated as the ratio of the number of foreground pixels correctly identified to the number of foreground pixels in the ground truth and precision is computed as the ratio of the number of foreground pixels correctly identified to the number of foreground pixels detected.

Figures 3 and 4 show that the proposed algorithm based on $S \alpha S$ distribution has slightly higher level of precision for the same values of the recall. The 


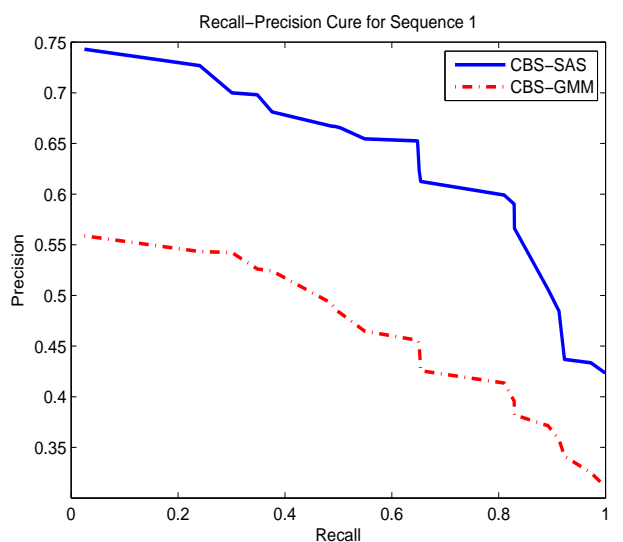

Figure 3: Recall-Precision curves of the CBS [24] and proposed CBS-S $\alpha S$ model, for sequence 1.

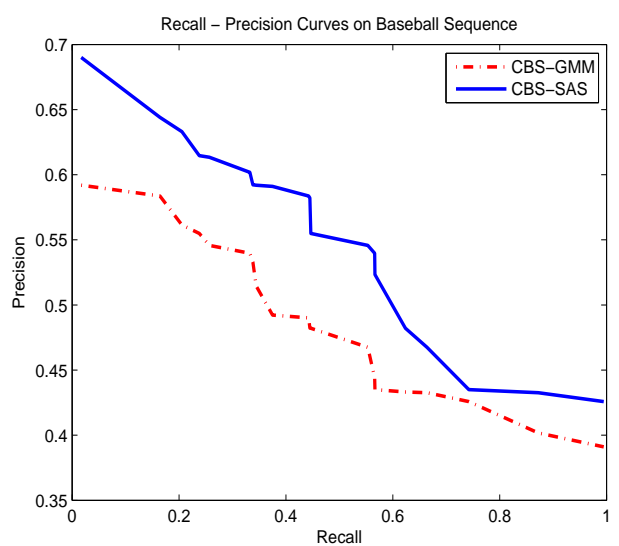

Figure 4: Recall-Precision curves of the CBS [24] and proposed CBS-S $\alpha S$ model, for sequence 2.

precision values directly relate to the number of correctly classified foreground pixels [4], and are inversely proportional to the misclassified foreground pixels. It is evident that compared with the pixel-based GMM [24] the proposed model maximises the proportion of correctly classified pixels and minimises the misclassification.

\section{Conclusions and Future Work}

This work shows the potential of the symmetric alpha stable distributions for background modeling in video sequences. The alpha stable distributions have the ability to handle heavy tails and thereby model movements in the background more efficiently than the models based on Gaussian assumptions.

The current research is focussed on the investigation of automatic detection of multiple people by using background subtraction with alpha stable distributions.
Acknowledgements. We acknowledge the support of the UK MOD Data and Information Fusion Defence Technology Centre under the Tracking Cluster project DIFDTC/CSIPC1/02 for supporting this research.

\section{References}

[1] H. Bhaskar, L. Mihaylova, and S. Maskell. Automatic target detection based on background modeling using adaptive cluster density estimation. In LNCS from the 3rd German Workshop on Sensor Data Fusion: Trends, Solutions, Applications, pages 130134, Universität Bremen, Germany, 24-28 Sept., 2007.

[2] S.-C. Cheung and C. Kamath. Robust techniques for background subtraction in urban traffic video. Video Communications and Image Processing, 5308(1):881-892, 2004.

[3] R. Cucchiara, C. Grana, M. Piccardi, and A. Prati. Detecting moving objects, ghosts and shadows in video streams. IEEE Trans. on Pattern Analysis and Machine Intel., 25(10):1337-1342, 2005.

[4] J. Davis and M. Goadrich. The relationship between precision-recall and ROC curves. In Proc. 23rd Intl. Conf. on Machine Learning, 2006.

[5] R. Duda, P. Hart, and D. Stork. Pattern Classification and Scene Analysis. John Wiley and Sons, Inc., 2000.

[6] A. Elgammal, R. Duraiswami, D. Harwood, and L. S. Davis. Background and foreground modeling using non-parametric kernel density estimation for visual surveillance. Proceedings of the IEEE, 90:1151-1163, July 2002.

[7] A. Elgammal, D. Harwood, and L. Davis. Non-parametric model for background subtraction. In Proc. 6th Europ. Conf. on Computer Vision, June/July 2000.

[8] B. Han, D. Comaniciu, Y. Zhu, and L. Davis. Incremental density approximation and kernel-based bayesian filtering for object tracking. In Proc. IEEE Conf. CVPR, 2004.

[9] O. Javed, K. Shafique, and M. Shah. A hierarchical approach to robust background subtraction using color and gradient informa- 
tion. In Proc. of IEEE Workshop on Motion and Video Computing, 2002.

[10] S M Kogon and D B Williams. On characteristic function based stable distribution parameter estimation techniques. In $\mathrm{R} J$ Adler, R E Feldman, and M S Taqqu, editors, A Practical Guide to Heavy Tails: Statistical Techniques and Applications. Birkhauser, Boston, MA, 1998.

[11] B.P.L. Lo and S.A. Velastin. Automatic congestion detection system for underground platforms. In Proc. of the 2001 International Symp. on Intelligent Multimedia, Video and Speech Processing, pages 158 - 161, 2001.

[12] X. Ma and C. Nikias. Parameter estimation and blind channel identification in impulsive signal environment. IEEE Tran. Sign. Proc., 43(12):2884-2897, Dec. 1995.

[13] Y. Ming, J. Jiang, and J. Ming. Background modeling and subtraction using a local-linear-dependence-based Cauchy statistical model. In In Proc. of the Seventh International Conf. on Digital Image Computing: Techniques and Applications, pages 469-478, 2003.

[14] N. Paragios and V. Ramesh. A MRF-based real-time approach for subway monitoring. In Proc. of IEEE Conf. CVPR, 2001.

[15] I. Pavlidis, V. Morellas, P. Tsiamyrtzis, and S. Harp. Urban surveillance systems: from the laboratory to the commercial world. Proceedings of the IEEE, 89(10):1478 -1497, 2001.

[16] D. Ramanan, D. A. Forsyth, and A. Zisserman. Tracking people by learning their appearance. IEEE Trans. Pattern Anal. Mach. Intell., 29(1):65-81, 2007.

[17] G. Samorodnitsky and M S Taqqu. Stable Non-Gaussian Random Processes: Stochastic Models with Infinite Variance. Chapman and Hall, New York, 1994.

[18] M. Shao and C. Nikias. Signal processing with fractional lower order moments: Stable processes and their applications. Proceedings of the IEEE, 81(7), July 1993.

[19] C. Stauffer and W. Grimson. Adaptive background mixture models for real-time tracking. In Proceedings of the CVPR, pages 246252, 1999.
[20] B. Stenger, V. Ramesh, N. Paragios, F. Coetzec, and J.M. Buhmann. Topology free hidden Markov models: application to background modeling. In Proc. of the Intl. Conf. on Computer Vision., 2001.

[21] K. Toyama, J. Krumm, B. Brumitt, and B. Meyers. Wallflower: Principles and practice of background maintenance. In Proc. Intl. Conf. Comp. Vision, pages 255-261, 1999.

[22] F. van der Heijden, R.P.W. Duin, and D. de Ridder. Classification, Parameter Estimation and State Estimation. John Wiley and Sons, 2004.

[23] J. Zhong and S. Sclaroff. Segmenting foreground objects from a dynamic textured background via a robust Kalman filter. In Proc. IEEE Intl. Conf. Computer Vis., 2003.

[24] Z. Zivkovic and F. van der Heijden. Efficient adaptive density estimation per image pixel for the task of background subtraction. Pattern Recognition Letters, 27(7):773-780, 2006.

[25] Z.Zivkovic. Improved adaptive Gausian mixture model for background subtraction. In Proc. of International Conf. Pattern Recognition, 2004. 